\title{
Remarks Concerning the Chemical Composition of the Atmosphere of Venus
}

\author{
By Hans E. Suess \\ University of California, San Diego, La Jolla, California \\ (Z. Naturforschg. 19 a, $84-87$ [1964] ; eingegangen am 24. Oktober 1963) \\ Dedicated to Prof. Dr. W. Groth on his sixtieth birthday
}

\begin{abstract}
It is still impossible to decide, on the basis of observational facts, whether the atmosphere of Venus is oxydizing, or reducing. Also, cosmochemical considerations do not lead to a conclusive answer to this important question. The atmosphere of Venus appears to be much more dense than that of the earth. For several reasons, it seems possible that the main constituent of this dense atmosphere is neon.
\end{abstract}

Enormous efforts have been made over the past years to solve the problems presented by our twin planet, Venus. Not much, however, has been added to the few relatively certain facts that we know about her. Size and mass of Venus are only slightly smaller than those of the earth; she has a thick atmosphere, much richer in carbon dioxide than our own, and a slow period of rotation, possibly synchronous to her orbital period around the sun. The main new findings are that the surface temperature seems to be much higher than previously expected. It is now estimated to be in excess of $700{ }^{\circ} \mathrm{K}^{1}$. The temperature of the dark side seems closely the same as that of the day side of the planet ${ }^{2}$. Radar observations indicate a retrograde period of about 240 days $^{3}$. Unexpected results were obtained by Murrey, Wildey, and Westphal ${ }^{4}$ who found, by using the 200-inch Mount Palomar telescope, that the temperatures above the cloud layer are lower in the areas of the poles than in the plane of the ecliptic. These results could be easily interpreted with the assumption of a more rapid rotation than that indicated by radar observations, but are difficult to understand if a slow period is assumed.

A strong microwave emission of the planet has been observed by ground stations, as well as by the Mariner space probe, and a number of models for

1 L. D. Kaplan, Tech. Report 32-379, Jet Prop. Lab., Dec. 1962 and private communication. - H. Spinrad, Publ. Astron. Soc. Pacific 74, 187 [1962].

2 F. Barath, A. Barrett, I. Copeland, D. James, and A. LilLEY, Science 139, 908 [1963].

3 R. M. Goldstein and R. L. Carpenter, Science 139, 911 [1963].

${ }^{4}$ B. D. Murrey, R. L. Wildex, and J. A. Westphal, J. Geophys. Res. 68, 4813 [1963]. the emisison of this radiation and for a corresponding structure of the atmosphere have been suggested $^{5}$. The most plausible of these models appears, at present, to be the so-called greenhouse model that assumes thermal emission from a hot ground level ${ }^{6}$. Other possibilities for an explanation of the microwave emission, however, such as one caused by electric dust storms ${ }^{7}$, or by photochemically induced chemical reactions ${ }^{8}$, cannot yet be ruled out with certainty.

The developments of the last years have produced enough additional information to furnish a basis for valid assumptions concerning temperatures, atmospheric pressures, and periods of rotation, but no progress has been made regarding the chemical composition of the atmosphere and the nature of the cloud cover of the planet. At first sight it may seem surprising that a planet, in many ways similar to the earth, should have an entirely different atmosphere. One can show, however, that the final composition of the atmosphere of an inner planet depends on many parameters in a very sensitive way ${ }^{9}$, so that small changes in the conditions under which the planet formed may have had very pronounced effects upon the chemistry of its atmosphere, which represents only a very small fraction of the order of $10^{-6}$ parts of its total mass.

5 C. S Sagan, Space Age Astronomy, Deutsch and Klemperer, Editors, Academic Press, New York and London 1962, p. 430.

6 C. SAGaN, Science 133, 849 [1961]; Icarus 1, 151 [1962].

7 Y. Mintz, Icarus 1, 172 [1962].

8 P. Harteck, priv. communication.

9 H. E. Suess, J. Geophys. Res. 67, 2029 [1962]. 
The early protoplanets, from which the planets formed, were presumably rich in hydrogen and helium. Earth and Venus are objects too small to retain these two light gases. Moreover, it can be shown that the main mass of these gases must have become separated from the non-volatile material before the planet had accumulated ${ }^{10}$. We have to assume that, in the case of the Earth, separation of the gases from the solids was nearly quantitative at a very early time, presumably long before the planet had obtained its final size. The present terrestrial atmosphere consists mainly of exhalation products - water, and some nitrogen - derived from the interior of the primitive earth. Photolysis of water and loss of hydrogen led to the formation of free oxygen ${ }^{11}$. Carbon dioxide, produced by oxidation of carbon, hydrocarbons, and other carbon-containing compounds, reacted with silicates to give free $\mathrm{SiO}_{2}$ and carbonates ${ }^{12}$, leaving only a small fraction in the atmosphere.

For the case of Venus, one might immediately expect a much higher rate of photolysis and of loss of hydrogen from the planet as a consequence of the higher temperature. This should have led to the formation of large amounts of free oxygen. It seems reasonable to assume that the amount of water contained in the silicates forming the planet was smaller than in the case of the earth. Still, with only one per cent of the water present on the surface of the young Venus, as compared to that of the present earth, a partial pressure of four atmospheres of free oxygen, or twenty times the amount now in the terrestrial atmosphere, should have formed if all the water vapor was photolyzed during geologic time. It seems, however, equally reasonable to assume that during the formation of Venus, decomposition of hydrocarbons may have led to the incorporation of larger amounts of elementary carbon into the surface rocks of Venus than into those of the earth ${ }^{9}$. Much, or all, of the oxygen formed by photolysis of water may have been consumed by this carbon, and the large amounts of $\mathrm{CO}_{2}$ in the atmosphere of Venus may thus be explained.

10 G. Kuiper, The Atmosphere of the Earth and the Planets, University of Chicago Press 1952, p. 324.

11 P. HARTeCK and H. J. D. Jensen, Z. Naturforschg. 3 a, 591 [1948]. - H. H. J. Poole, Nature, Lond. 166, 761 [1950]. 12 H. C. UREY, Handbuch d. Physik 52, 363 [1959].

13 V. K. Prokofjev and N. N. Petrova, Mem. Soc. Roy. Sci., Liège 7, 311 [1962].
The Russian astronomers, Prokofjev and PETRova ${ }^{13}$, report the observations of relatively strong lines in the spectrum of Venus that can be interpreted as $\mathrm{O}_{2}$ lines displaced by Dopplen shift due to the relative motion of earth and Venus. Other workers question such an interpretation ${ }^{14}$. The presence of $\mathrm{H}_{2} \mathrm{O}$ would make it appear probable that oxygen is present. So far, only upper limits for the amounts of water vapor have been given, equal to approximately one-tenth the amount in the terrestrial atmosphere ${ }^{4,15}$. No evidence exists for a reducing atmosphere. Carbon monoxide should be easily observable if amounts of this gas were present corresponding to thermodynamic equilibrium with $\mathrm{CO}_{2}$ and carbon at temperatures above $700^{\circ}$. No indication for this has been found.

As there is presently no way to decide between a reducing or an oxydizing atmosphere, it is also not possible to answer the question of the chemical nature of the clouds that cover Venus. A reducing atmosphere, combined with a surface temperature in excess of $700{ }^{\circ} \mathrm{K}$, will almost certainly produce thick clouds of sulphur vapor, and also more tenuous clouds of carbon disulphide, and many other sulphur-containing compounds. Also, $\mathrm{C}_{2} \mathrm{O}_{3}$ has been suggested as a possible source for clouds in a reducing atmosphere ${ }^{16}$. The presence of water, hydrocarbons, and other hydrogen-containing compounds can be excluded with certainty, because under reducing conditions such compounds react at $700{ }^{\circ} \mathrm{K}$ to give an equilibrium partial pressure of hydrogen which rapidly leaves a planet of the size of the earth or Venus.

If one assumes the existence of an oxydizing atmosphere, then the presence of the clouds is even more difficult to interpret. If water were still present, the clouds could certainly be water, continuously supplied from the interior. Many inorganic compounds are volatile at temperatures of $700{ }^{\circ} \mathrm{K}$, in particular, halogenids, which should cover the surface of a dry planet in large amounts. Oxides of metals, such as $\mathrm{Zn}, \mathrm{Cd}$, and others, also show an appreciable vapor pressure at this temperature.

14 L. Kaplan, private communication.

15 H. Spinrad, Icarus 1, 266 [1962].

16 P. Harteck, private communication. For possibilities of other photochemically produced substances, see W. GRотн, Z. Elektrochem. 45, 262 [1939]. 
Detailed investigations of the conditions under which a greenhouse effect would produce temperatures in excess of $700{ }^{\circ} \mathrm{K}$, show that besides $\mathrm{CO}_{2}$, large amounts of an inert gas have to be present, in addition to compounds that absorb in the infrared. The main difficulty presented by the extreme greenhouse model, however, is not the necessity of an atmosphere sufficiently opaque in the infrared, but of an atmosphere that is, at the same time, sufficiently transparent in the visible, in spite of the presence of vapors of many volatiles, as well as dust. The opacity in the visible puts an upper limit on the total pressure of the atmosphere that can contribute to greenhouse heating of the planetary surface.

Furthermore, the question of the origin of large amounts of an inert gas, usually taken to be nitrogen, remains to be investigated. It will be shown in the following that it is more probable that neon is the main constituent of a dense atmosphere of Venus, rather than nitrogen.

The cosmic abundance of neon was first determined in a quantitative way by UNsöLD ${ }^{17}$, by spectral analysis of the B-Star, $\tau$-Scorpii. UnsöLD gave a neon abundance slightly higher than that of oxygen. Later on, Traving ${ }^{18}$ revised this figure and estimated the neon abundance to be about 0.4 times that of oxygen. This value was adopted by Suess and UREY ${ }^{19}$ in their tables. From neon determinations of several more hot B-stars that show Ne-lines, Aller ${ }^{20}$ now estimated the most probable value of the neon abundance to be about 0.9 times that of oxygen. Such an abundance value for neon agrees approximately with the amounts of neon in gas-rich meteorites ${ }^{21}$ which, in most cases, show a $\mathrm{He}^{4} / \mathrm{Ne}^{20}$ ratio of about 320 . It is possible that this ratio was somewhat changed by diffusive loss from the meteorites, but detailed consideration of the relative amounts of the rare gases and their isotopes in gasrich meteorites, show that a primoridal $\mathrm{He} / \mathrm{Ne}$ ratio of more than 800 is highly improbable ${ }^{22}$. We, therefore, have to conclude that neon was a very abun-

17 A. UNsöLD, Z. Astrophys. 21, 1, 22 [1941] ; 21, 229 [1942] ; 23, 75, 100 [1944].

18 G. Traving, Z. Astrophys. 36, 1 [1955]; 41, 215 [1957].

19 H. E. Suess and H. C. Urey, Revs. Mod. Phys. 28, 53 [1956].

${ }^{20}$ L. H. Aller, The Abundance of the Elements, Interscience Publish. Inc., New York 1961 and private communications.

21 P. Signer and H. E. Suess, Earth Sciences and Meteoritics, Geiss and Goldberg, Editors, North Holland Publ., Amsterdam 1963, p. 240. dant gas in the primordial solar nebula from which the planets formed.

In the case of the earth, the fraction of rare gases originally associated with its matter, and now present in its atmosphere, ranges from $10^{-11}$ for neon to $10^{-7}$ for the heavy gases, krypton and xenon ${ }^{23}$. The amount of neon present represents an upper limit for the fraction of solar matter retained by the primitive earth, because some or all of this amount could have been originally retained by solids and could have been added to the atmosphere through degassing of the earth.

An interesting recent article by CAMERoN ${ }^{23}$ suggests that the fraction of the primordial solar nebula retained by Venus was much greater than that retained by the earth. Cameron suspects that the reason for this difference is connected with the fact that the earth has a moon and Venus has none. In a way, CAMERon revives an early idea of the writer ${ }^{24}$ that the essentially complete loss of the atmosphere of the earth was caused by rapid rotation during the latter stages of the formation of the planet. This rotation led to the spinning-off of the atmosphere and of material that formed the moon. Cameron considers the possibility of a connection with a catastrophic formation of the core of the earth, according to Elsasser's eccentric theory ${ }^{25}$. He suggests that the same catastrophic event was also responsible for the loss of the primordial atmosphere and for the formation of the moon.

Independently from the validity of such considerations, the idea appears very attractive that Venus has retained a much larger fraction of the primitive solar nebula than the less than $10^{-11}$ parts retained by the earth.

Table 1 shows the composition of a planetary atmosphere derived entirely from solar material after loss of hydrogen and helium. The values are based on the most recent determinations of element abundances as given by Aller ${ }^{26}$. Because of the uncertainties in our knowledge of these values, about a factor of two in most cases, it is impossible to pre-

22 H. E. Suess, H. Wänke, and F. Wlotzka, Geochim. Cosmochim. Acta, in press.

23 A. Cameron, Icarus 2, 249 [1963].

${ }^{24}$ H. E. Suess, J. Geol. 57, 600 [1949].

25 W. Elsasser, in Earth Sience and Meteoritics, Geiss and Goldberg, Editors, North Holland Publ. Amsterdam 1963.

26 L. Aller, private communication. 


\begin{tabular}{|c|c|c|c|c|}
\hline Element & $\begin{array}{l}\log _{10} \mathrm{~N} \\
\text { (abun- } \\
\text { dance } \\
\text { units) }\end{array}$ & $\begin{array}{c}\text { atoms per } \\
\text { atom Si }\end{array}$ & \multicolumn{2}{|c|}{$\begin{array}{c}\text { Relative number } \\
\text { of gas molecules } \\
\text { after loss of } \mathrm{H} \\
\text { and } \mathrm{He}\end{array}$} \\
\hline $\mathrm{H}$ & 10.50 & 30,000 & 一 & - \\
\hline $\mathrm{He}$ & 9.76 & 4,700 & - & - \\
\hline $\mathrm{C}$ & 7.00 & 10 & $\mathrm{CO}_{2}$ & 10 \\
\hline $\mathrm{N}$ & 6,50 & 3 & $\mathrm{~N}_{2}$ & 1.5 \\
\hline 0 & 7.45 & 30 & $\mathrm{O}_{2}$ & $0 \ldots 2$ \\
\hline $\mathrm{Ne}$ & 7.40 & $5 \ldots 25$ & $\mathrm{Ne}$ & $5 \ldots 25$ \\
\hline $\mathrm{A}^{36}$ & 5.18 & 0.15 & A & 0.15 \\
\hline $\begin{array}{l}\text { Si and } \\
\text { Metals }\end{array}$ & 6.5 & 3 & - & - \\
\hline $\mathrm{S}$ & 5.6 & 0.4 & $\mathrm{SO}_{2}$ & 0.4 \\
\hline
\end{tabular}

Table 1. Composition of solar matter according to ALLer ${ }^{20}$ and composition of gas phase after loss of hydrogen and helium.

dict whether or not free oxygen will form in such a medium, because the main fraction of oxygen will combine with carbon to form $\mathrm{CO}_{2}$, and about $25 \%$ of it will form oxides of silicon, of sulphur, and of metals. The relative amount of carbon dioxide listed in the table should be considered an upper limit, because of the possibility of reactions with the surface rocks that consume $\mathrm{CO}_{2}$ as well as $\mathrm{SO}_{2}$. The amount of neon in such an atmosphere will be between 3 and 15 times that of $\mathrm{N}_{2}$, if the $\mathrm{N}_{2}$ obtained by degassing of the planet can be neglected.

It appears quite probable that the Cytherean atmosphere contains more than $50 \%$, and possibly as much as $85 \%$, of neon. Clearly, a large content of a mono-atomic noble gas considerably changes the scale-height, adiabatic lapse rate, and other parameters used in providing a consistent model atmosphere for Venus. Also, a dry, neon-rich atmosphere may well provide unexpected possibilities for nonthermal microwave emission. Re-evaluation of the observational facts, from the point of view of a possibly high neon content, may well lead to considerably different results than those considered acceptable at present. It might even be premature to exclude every possibility of finding an atmosphere on Venus that is, at some level of its profile, breathable by humans.

\section{A cknowledgement}

Fiancial support for this study was provided by the National Aeronautics and Space Administration, through Grant No. NsG-322. 